\title{
Chemical tuning of NaSICON surfaces for fast-charging Na metal solid-state batteries
}

\author{
Edouard Quérel, 1,* leuan D. Seymour,' Andrea Cavallaro, ${ }^{1}$ Qianli Ma, ${ }^{2, * *}$ Frank Tietz, ${ }^{2}$ Ainara \\ Aguadero, ${ }^{1, * * *}$ \\ 'Department of Materials, Imperial College London, London, SW7 2AZ, UK \\ 2Forschungszentrum Jülich $\mathrm{GmbH}$, Institute of Energy and Climate Research, Materials Synthesis and Processing \\ (IEK-1), 52425 Jülich, Germany \\ *Correspondence: edouard.querel16@imperial.ac.uk \\ ${ }^{* *}$ Correspondence: q.ma@fz-juelich.de \\ ***Correspondence: a.aguadero@imperial.ac.uk
}

\section{ABSTRACT}

Solid-state batteries (SSBs) with alkali metal anodes hold great promise as energetically dense and safe alternatives to conventional Li-ion cells. Whilst, in principle, SSBs have the additional advantage of offering virtually unlimited plating current densities, fast charges have so far only been achieved through sophisticated interface engineering strategies. Here, we reveal that such interface engineering can be easily achieved by tuning the chemistry of NaSICON solid electrolytes $\left(\mathrm{Na}_{3.4} \mathrm{Zr}_{2} \mathrm{Si}_{2.4} \mathrm{P}_{0.6} \mathrm{O}_{12}\right)$ and taking advantage of the thermodynamic stabilization of $\mathrm{Na}_{3} \mathrm{PO}_{4}$ layer on their surface upon thermal activation. The optimized planar $\mathrm{Na|NZSP}$ interfaces are characterized by their exceptionally low interface resistances (down to $0.1 \Omega \mathrm{cm}^{2}$ at room temperature) and, more importantly, by their tolerance to large plating current densities (up to $10 \mathrm{~mA} \mathrm{~cm}$. ${ }^{2}$ ) even for extended cycling periods of 30 minutes (corresponding to an areal capacity $5 \mathrm{mAh} \mathrm{cm}^{-2}$ ).

\section{INTRODUCTION}

Increasing the energy density and enhancing the safety of batteries are two notorious objectives motivating the research for new cell chemistries. ${ }^{1,2}$ Replacing conventional intercalation type anodes by alkali metal ones could offer a significant increase in energy density thanks to the high specific capacity of alkali metals $(3,860$ $\mathrm{mAh} \mathrm{g}^{-1}$ for $\mathrm{Li}$ and 1,166 $\mathrm{mAh}^{-1}$ for $\mathrm{Na}$ ) and their low redox potential (-3.04 $\mathrm{V}$ and $-2.71 \mathrm{~V}$ versus SHE standard hydrogen electrode - for $\mathrm{Li}$ and $\mathrm{Na}$, respectively). However, to this day, the use of alkali metal anodes in batteries employing liquid electrolytes has been confronted to challenging problems of dendrite growths and low coulombic efficiency. ${ }^{3,4}$ Alternatively, solid electrolytes (SEs) used in solid-state batteries (SSBs) enable, in theory, the use of alkali metal anodes by making the electrolyte impenetrable to dendrites. SEs also improve cells' safety by removing the flammability risks associated with organic liquid electrolytes.

As the environmental impact of $\mathrm{Li}$-ion cells is becoming more and more criticized, ${ }^{5-9}$ new battery chemistries are also required to be more sustainable in addition to being more electrochemically performant. SSBs using $\mathrm{Na}^{+}$ions as charge carriers aim to provide such a sustainable and economical alternative. Furthermore, if the high specific capacity of Li metal could initially make one think that Li-SSBs are more advantageous in terms 
of gravimetric and volumetric energy density, recent models including all the active and inactive components of a cell indicate that some Na-SSBs could compete with their Li counterparts. ${ }^{10}$

Among suitable SEs for Na-SSBs, the family of $\mathrm{Na}^{+}$Superlonic CONductors (NaSICON) of composition $\mathrm{Na}_{1+\times} \mathrm{Zr}_{2} \mathrm{Si}_{x} \mathrm{P}_{3-x} \mathrm{O}_{12}(0 \leq x \leq 3)$ has long been recognized for its high ionic conductivity and convenient processability in air. ${ }^{11,12} \mathrm{~A}$ renewed interest around the synthesis of these materials has recently contributed to enhance their performance to an unprecedented total room temperature conductivity of $5 \mathrm{mS} \mathrm{cm}^{-1}$ (15 mS $\mathrm{cm}^{-1}$ bulk conductivity) for the $\mathrm{Na}_{3.4} \mathrm{Zr}_{2} \mathrm{Si}_{2.4} \mathrm{P}_{0.6} \mathrm{O}_{12}$ composition (further referred to as NZSP). ${ }^{13}$ Improvements in the total ionic conductivity of NaSICON materials were obtained from the optimization of the bulk phase but also, to a large extent, by controlling the densification of these polycrystalline materials. ${ }^{14-16}$ Slight offstoichiometric excesses in $\mathrm{Na}$ and $\mathrm{P}$ constitute a chemical tuning strategy which has led to highly dense NZSP. ${ }^{14,15,17}$

Although good bulk properties are an essential prerequisite for a material to be considered as a suitable SE, important limitations to the electrochemical performance of SSBs are known to emerge from issues at the anode|SE interface, even more when fast cycling rates are targeted (in the order of several $\mathrm{mA} \mathrm{cm}^{-2}$ ). ${ }^{18}$ Some interrelated challenges affecting the anode|SE interface include: large interface resistances; ${ }^{19}$ the coalescence of pores during stripping (discharge) $;^{18,20}$ inhomogeneous plating (charge) with the risk of a short-circuit if the SE is penetrated by alkali metal filaments. ${ }^{21}$ Several sophisticated interface engineering strategies have been suggested to solve these problems, such as: improving interfacial wetting by introducing thin alkaliphilic interlayers; ${ }^{22}$ the application of large stack pressures to prevent contact loss during cycling;23-25 the use of 3D interfaces to increase the surface-active area and mitigate contact loss. ${ }^{26}$ Unoptimized Na|NaSICON interfaces are also affected by these issues with, for instance, interface resistances in the $\mathrm{k} \Omega \mathrm{cm}^{2}$ range still often reported (see Supplementary Table 1).

In this study, we reveal that fast-plating rates can be achieved without expensive interface engineering for NZSP electrolytes by taking advantage of the in-situ stabilization of a $\mathrm{Na}_{3} \mathrm{PO}_{4}$ layer on their surface upon thermal activation. We demonstrate the existence of this $\mathrm{Na}_{3} \mathrm{PO}_{4}$ layer using $\mathrm{X}$-ray photoelectron spectroscopy (XPS), low energy ion scattering (LEIS) and first principles calculations based on density functional theory (DFT). We report that a negligible Na|NZSP interface resistance (down to $0.1 \Omega \mathrm{cm}^{2}$ ) can be systematically obtained with thermally activated NZSP samples. As revealed by a critical current density of $10 \mathrm{~mA} \mathrm{~cm}^{-2}$, the optimization of the Na|NZSP interface described in this study paves the way for fast charging Na-SSBs.

\section{RESULTS}

\section{The processing of NZSP surfaces impacts the Na|NZSP interface resistance}

To evaluate how the NZSP surface processing influences the electrochemical performances of Na|NZSP interfaces, impedance spectra of three types of Na|NZSP|Na symmetrical cells are compared. The surface of NZSP pellets used for these cells are either unaltered after sintering (NZSP as sintered), roughly polished with ethanol as solvent (NZSPEtOH500), or polished and annealed (NZSPEtOH500-anneal). For more information about the samples' nomenclature, the reader is referred to the Experimental Section. Normalized Nyquist plots 
representing the impedance spectra of the three symmetrical cells are presented in Figure 1. The models used for equivalent circuit fitting are included in inset and consist of a resistor $R_{H F}$ (high frequency resistance), an inductor $L$ (accounting for high frequency inductive effects from the measurement setup), and either one or two R-CPE elements (CPE: constant phase element) depending on the number of observed semi-circles. The calculated fitting parameters can be found in Table S2.

Detecting the bulk diffusion of $\mathrm{Na}^{+}$ions through the NSZP structure is not possible at room temperature in the frequency range covered by the frequency response analyzers used in this study. ${ }^{13}$ In the equivalent circuits, $\mathrm{R}_{\mathrm{HF}}$ therefore accounts for the resistive contributions of the bulk diffusion with the ohmic losses of the impedance analyzer. For all three samples, the semi-circle observed at high frequency (with an apex at a frequency of around $1 \mathrm{MHz}$ and capacitance of $\mathrm{C}_{\mathrm{GB}} \approx 3-5 \times 10^{-9} \mathrm{~F}$ ) can be attributed to grain boundary diffusion, as was already demonstrated in previous literature and confirmed here with the impedance results of a Au|NZSP|Au symmetrical cell (see Supplementary Fig.2). ${ }^{13,27}$

A second semi-circle attributed to the transfer of $\mathrm{Na}^{+}$ions across the Na|NZSP interface (modelled by $\mathrm{R}_{\text {int }}$ and $\mathrm{CPE}_{\text {int, }}$ equivalent capacitance $\mathrm{C}_{\text {int }}=6.99 \times 10^{-7} \mathrm{~F}$ ) can be clearly observed for the NZSPEtOH500 sample. The calculated interface resistance of $24.2 \Omega \mathrm{cm}^{2}$ is quite low in comparison to other studies reported in Table $\mathrm{S} 1$. As an interface semi-circle is not distinguishable for the NZSP as sintered and NZSPEtOH500-anneal samples, their interface resistances were estimated using the procedure described by Krauskopf et al. ${ }^{28}$ by calculating the difference between $Z^{\prime}$ data $\left(f_{\min }\right)$ and $Z_{\text {fit }}^{\prime}(f \rightarrow 0 \mathrm{~Hz})$. This results in interfacial resistances as low as 0.2 and 0.1 $\Omega \mathrm{cm}^{2}$ for the $\mathrm{NZSP}_{\text {as sintered }}$ and NZSPEtOH500-anneal samples, respectively.

To rule out a possible influence of the polishing medium in the larger interface resistance of NZSPEtOH500, another set of symmetrical cells were assembled from dry polished samples (NZSP dry500). The interface resistance calculated from the NZSP dry500 cell presented in Supplementary Fig.3 $\left(22.1 \Omega \mathrm{cm}^{2}\right)$ is equivalent to NZSPEtOH500, indicating that the use of a polishing solvent has no influence on the Na|NZSP interface resistance.

A much more significant impact on the Na|NZSP interface resistance is observed when NZSP samples are

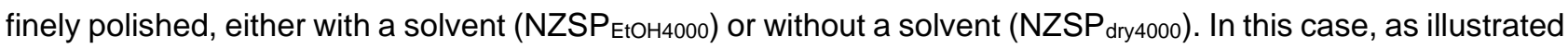
in Supplementary Fig.3 and Supplementary Fig.4, interface resistances in the range of $4-9 \mathrm{k} \Omega \mathrm{cm}^{2}$ are obtained. Yet, even if NZSP samples are finely polished, a thermal annealing can restore a negligible Na|NZSP interface resistance (see Supplementary Fig.3 and Supplementary Fig.4).

These results demonstrate that the Na|NZSP interface resistance depends on the processing conditions of the NZSP surface. Thermally activated NZSP surfaces yield the best electrochemical performance. The detrimental effect of polishing NZSP surfaces on the Na|NZSP interface resistance can be cancelled by annealing. 


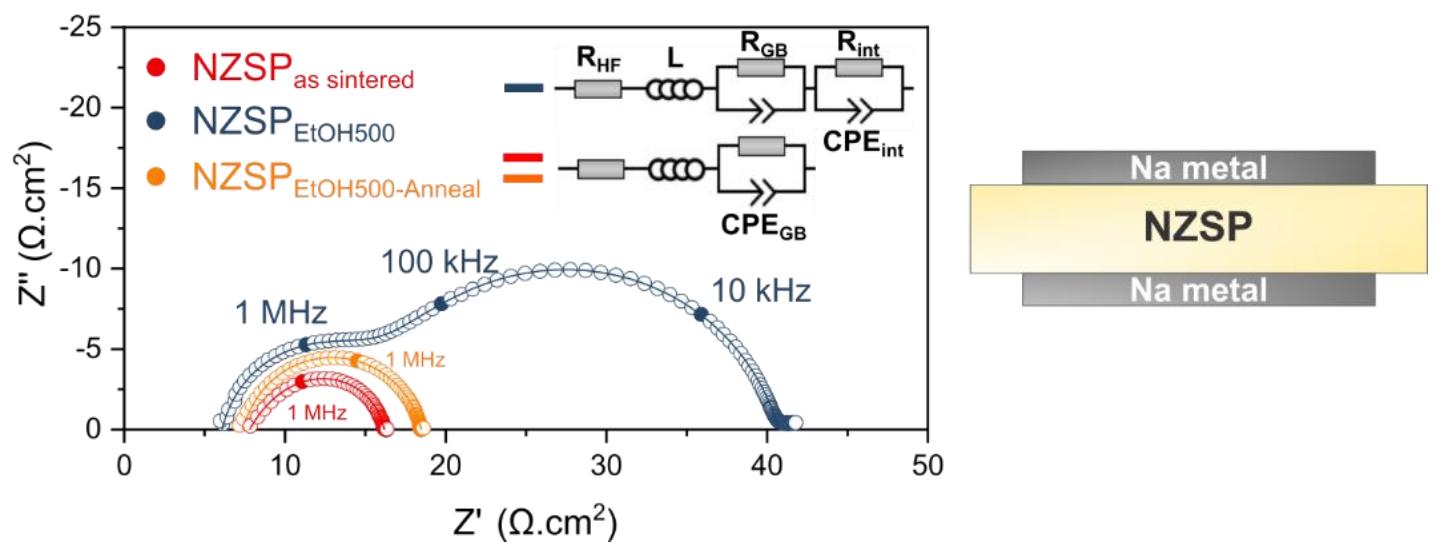

Figure 1. The processing of NZSP surfaces impacts the Na|NZSP interface resistance Nyquist plots of Na|NZSP|Na symmetrical cells (NZSP as sintered, NZSPEtOH500 and NZSPEtOH500-anneal). Data points are shown as open circles, frequency markers are shown as filled circles, and the respective fits (obtained with the equivalent circuits shown in inset) are represented as a solid line. The data presented are normalized to the area of the electrodes and divided by two.

\section{Comparison of the surface chemistry of NZSP samples by XPS}

To elucidate the origin of the good electrochemical performance of cells employing thermally activated NZSP pellets, the surface composition of NZSP samples were first analyzed by XPS. Figure 2 compares the signals of three NZSP core levels (Na1s, P2p and C1s) for three samples (NZSP as sintered, NZSPEtOH500 and NZSPEtOH500anneal). The $\mathrm{Zr} \mathrm{d}_{5 / 2}$ peak area was used as a normalization constant to compare the relative signal intensities between samples. Survey spectra and other core level spectra can be found in Supplementary Fig.5 and Supplementary Fig.6.

C1s spectra were collected for each sample to monitor the extent of surface contamination. Although the presence of adventitious carbon species on the surface of SE samples has frequently been linked to poor ionic transport across metal|SE interfaces, ${ }^{19,29-31}$ Figure 5 reveals that NZSP samples, whichever surface processing they have received, have a similarly low level of surface carbon contamination. The only exception is for the finely polished NZSP samples (NZSPEtOH4000 and NZSP dry4000) for which a higher level of $\mathrm{C}$ contamination was detected (see Supplementary Fig.7 and Supplementary Fig.8).

As can be seen from Figure 2, the Na1s and P2p signals of the thermally activated samples (NZSP as sintered

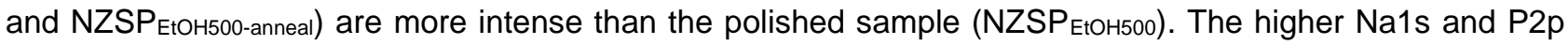
intensities indicate a higher concentration of these two elements on the surface of thermally activated samples which could be explained by the stabilization of a thin sodium phosphate layer on their surface. The Na1s and P2p binding energies of this new layer are unfortunately overlapping with the NZSP signals.

The higher $\mathrm{Na}$ and $\mathrm{P}$ concentration on the thermally activated NZSP surfaces is also verified with finely polished samples as illustrated in Supplementary Fig.7. Here again, the thermally activated sample (NZSPEtOH4000-anneal), which was characterized by a low interface resistance, has more intense Na1s and P2p signals than the polished sample (NZSPEtOH4000). 


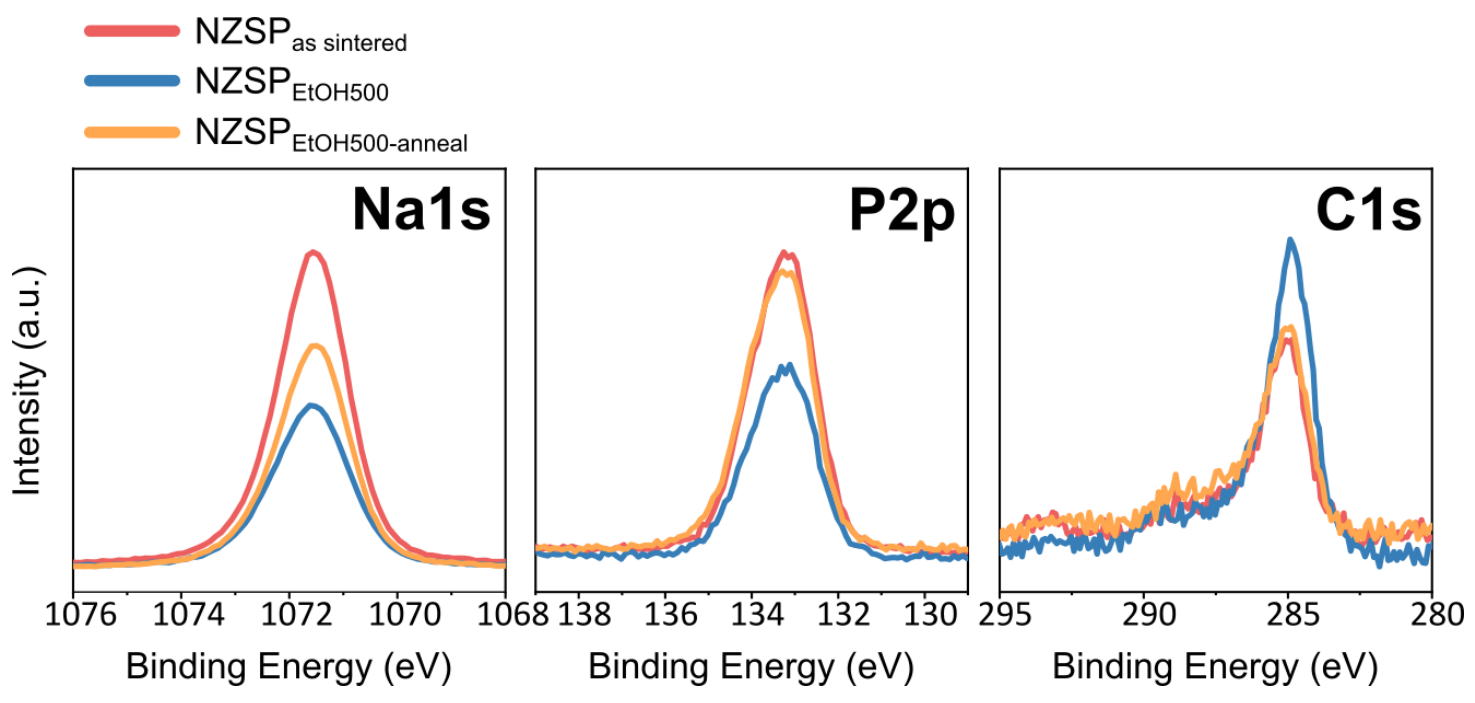

Figure 2. Comparison of the surface chemistry of NZSP samples by XPS Core level XPS spectra of samples NZSP as sintered, NZSPEtOH500 and NZSPEtOH500-anneal. (charge correction: Zr3d5/2 at $182.4 \mathrm{eV}$; intensity normalization between samples: Zr3d5/2 peak area).

\section{LEIS reveals the surface composition of thermally activated NZSP}

To confirm the composition and determine the coverage of the surface layer whose existence was suggested by XPS, LEIS was employed. LEIS relies on the binary collision between low energy primary ions (here $\mathrm{He}^{+}$ ions) and the elements present on the surface of a specimen. A LEIS instrument collects the $\mathrm{He}^{+}$ions scattered by a surface at an optimum angle defined by the LEIS detector. The position of the peaks observed in the LEIS energy spectra depends on the primary ions mass, the primary ions energy, the mass of the analyzed atomic species and the detector acceptance angle. To simplify the syntax in the following paragraphs, the peaks will be named according to the analyzed interacting element (e.g. "the Na peak") although it should be understood as scattered $\mathrm{He}^{+}$ions which interacted with the respective surface atom. As the method employs low energy and low mass primary ions, LEIS is a non-destructive technique able to measure the chemical composition of the uppermost atomic layer of the specimen (Supplementary Fig.9). In practice, as with any ion analysis technique, some partial sputtering of surface atoms will occur and is a function of the primary ion dose exposure. For all the LEIS analyses, the $\mathrm{He}^{+}$ion primary beam was scanned on a large area of $500 \times 500 \mu \mathrm{m}$. The surface composition determined by LEIS is an average composition of this entire area.

Two LEIS energy spectra corresponding to the surface (in red) and the bulk (in blue) composition of a NZSP as sintered sample are presented in Figure 3 (a). The surface spectrum was collected with a $\mathrm{He}^{+}$ion dose of $8 \times 10^{14}$ ions $\mathrm{cm}^{-2}$. After having collected this surface spectrum, an $\mathrm{Ar}^{+}$ion sputtering beam was used to reveal deeper layers of the sample. At regular intervals, the $\mathrm{Ar}^{+}$sputtering beam was alternated with the $\mathrm{He}^{+}$ion analysis beam to obtain a depth profile. The averaged signal of all the spectra collected during this depth profile is the bulk spectrum in Figure 3(a). The LEIS energy spectrum of the NZSP as sintered first atomic layer indicates that it 


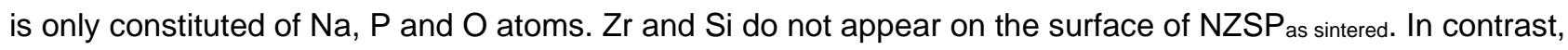
the averaged depth profile LEIS energy spectrum (in blue) shows that $\mathrm{O}, \mathrm{Na}, \mathrm{Si}, \mathrm{P}$ and $\mathrm{Zr}$ are present underneath a thin surface layer. Regarding the Na peak, the surface of NZSPas sintered samples seems to be enriched in sodium in comparison to its bulk. In reality, the low intensity of the Na peak in the bulk spectrum is associated to a challenging issue of preferential sputtering (see Experimental Section for more details).

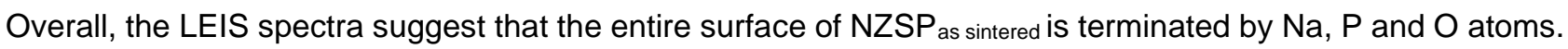
The presence of a $\mathrm{Zr}$ in-depth signal around $2500 \mathrm{eV}$ in the LEIS energy spectrum of the first atomic layer (see Experimental Section for more details) indicates that the sodium phosphate surface layer must be extremely thin.

In Figure 3(b), the surface composition of $\mathrm{NZSP}_{\text {as }}$ sintered is compared with the composition of a polished

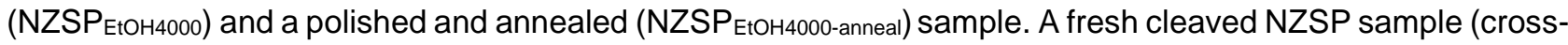
section of a pellet) was also analyzed. This measurement provided information about the bulk composition of NZSP samples. For each sample, LEIS spectra were collected on three different areas. On each area, four consecutive measurements were collected. A schematic illustrating the sequential LEIS analysis is presented in Supplementary Fig.10. In Figure 3(b), the averaged $\mathrm{Na}, \mathrm{Zr}, \mathrm{Si}, \mathrm{P}$ and $\mathrm{O}$ peak normalized intensities are plotted as a function of the $\mathrm{He}^{+}$primary ion dose. Details about the normalization procedure can be found in the Experimental Section.

Figure 3(b) can be used to compare, the surface composition of the different NZSP samples at a given ion dose. The largest differences can be found for the lowest $\mathrm{He}^{+}$ion dose $\left(1.6 \times 10^{15}\right.$ ions $\mathrm{cm}^{-2}$, shown in dark red). Thermally activated NZSP samples have more $\mathrm{Na}$ and $\mathrm{P}$ on their surface than polished samples. The $\mathrm{Na}$ and $\mathrm{P}$ normalized peak areas of $\mathrm{NZSP}_{\text {as sintered }}$ are more than twice as high as the NZSPEtOH4000 sample. More importantly, the signal corresponding to Si cannot be initially detected on the surface of NZSP as sintered samples and only appears after a cumulated ion dose of $4.8 \times 10^{15}$ ions $\mathrm{cm}^{-2}$.

Figure 3(b) also shows that the surface composition of thermally activated samples is more impacted by the received ion dose than the polished sample. As the ion dose increases from 1.6 to $6.4 \times 10^{15}$ ions $\mathrm{cm}^{-2}$ and deeper atomic layers are slowly revealed, differences in the surface chemical composition of samples attenuate. Taking NZSPas sintered as an example, the intensity of the $\mathrm{P}$ peak attenuates with an increasing ion dose, while simultaneously the intensity of the $\mathrm{Zr}$ and Si peaks increases. Variations in the intensity of the $\mathrm{Na}$ peak are not related to changes in composition but rather to the previously mentioned problem of preferential sputtering; this explains why the intensity of the Na peak decreases for all samples with higher ion doses. In

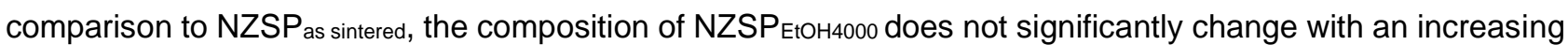
ion dose and stays within its error bars. This suggest that the $\mathrm{Na}_{3} \mathrm{PO}_{4}$ layer on the surface of thermally activated NZSP samples is only a few nanometers thick. By removing this surface layer with a low ion dose, a surface composition close to that of a polished sample is obtained. LEIS shows that the entire NZSP surface is covered by a thin $\mathrm{Na}_{3} \mathrm{PO}_{4}$ layer. Locally, this layer was also observed to be thicker by scanning electron microscopy (Supplementary note 1, Supplementary Fig.11-14). 
In agreement with our XPS results, our LEIS analysis confirms that thermally activated samples stabilize a very thin sodium phosphate layer on their surface.

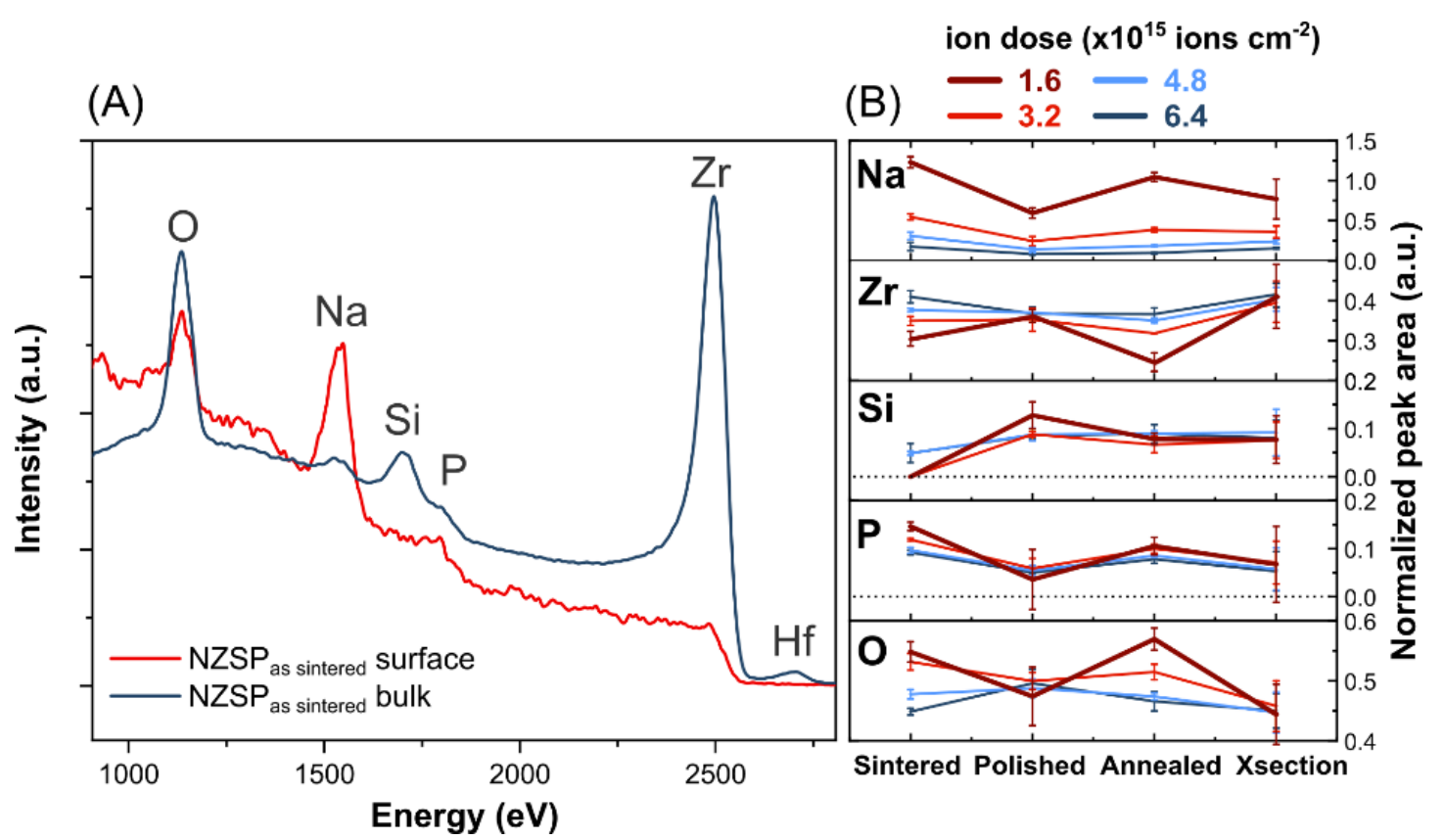

Figure 3. LEIS reveals the surface composition of thermally activated NZSP

(A) ${ }^{4} \mathrm{He}^{+}$ion LEIS energy spectra of a NZSP as sintered sample comparing the signal of its first atomic layer (for a $8 \times 10^{14}$ ions $\mathrm{cm}^{-2}$ dose, in red) to its average bulk signal (in blue), (B) Comparison of the normalized LEIS peak areas of four NZSP samples with increasing ion doses ("Sintered"=NZSP as sintered, "Polished"=NZSPEtOH4000, "Annealed"=NZSPEtOH4000anneal, "Xsection"=NZSP cross-section). Each point is the average of three peak areas measured on three different points on the surface of each sample. Error bars correspond to standard deviations.

\section{DFT calculations predict a lower surface energy for $\mathrm{Na}_{3} \mathrm{PO}_{4}$ terminated NZS/NZP}

As our experimental results converge to the conclusion that the thermal activation of NZSP samples results in the stabilization of a sodium phosphate surface, we used DFT calculations to understand the thermodynamic driving force for this process. The aim of this section is to compare the energies of $\mathrm{Na}_{3} \mathrm{PO}_{4}$ terminated NaSICON surfaces with that of bare NaSICON surfaces. In the absence of an extensive DFT literature on the $\mathrm{Na}_{1+x} \mathrm{Zr}_{2} \mathrm{Si}_{x} \mathrm{P}_{3-\mathrm{x}} \mathrm{O}_{12}(0 \leq \mathrm{x} \leq 3)$ family of materials, a first necessary step was to identify the NZSP surfaces with lowest energy (i.e. the most likely to form experimentally). A decision was made to focus on the two end member compositions $\mathrm{NaZr}_{2} \mathrm{P}_{3} \mathrm{O}_{12}$ (NZP) and $\mathrm{Na}_{4} \mathrm{Zr}_{2} \mathrm{Si}_{3} \mathrm{O}_{12}$ (NZS) to remove the complexity of handling partial

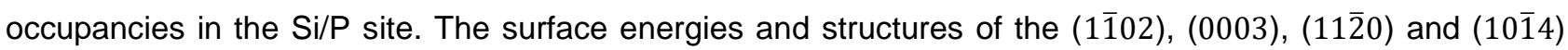
planes of NZP and NZS are shown in Table 1, Supplementary Table 3 and Supplementary Fig. 15.

Previous studies of phosphates and oxides have demonstrated that the cleavage of transition metal-O bonds results in high energy surfaces. ${ }^{32,33}$ For NZP and NZS, the (1102) surface has the lowest energy, which is consistent with the fivefold coordination of $\mathrm{Zr}\left(\mathrm{ZrO}_{5}\right)$ on the surface in comparison to the (1014) and (0003), planes with four- and threefold coordinated $\mathrm{Zr}$, respectively. The $(11 \overline{2} 0)$ surface, which also has fivefold 
coordinated $\mathrm{Zr}$ on the surface, has a higher surface energy than the (1102) plane as the surface $\mathrm{PO}_{4} / \mathrm{SiO}_{4}$ groups have a lower coordination with neighboring $\mathrm{Na}^{+}$ions. Although only a limited number of surface terminations have been investigated in this study, the significantly lower energy of the $\{1 \overline{1} 02\}$ family of planes relative to other surfaces for NZP/NZS would result in an approximately cubic morphology from a Wulff construction (Figure 4), which is consistent with the cubic morphology of grains observed experimentally in this work and analogous NaSICON materials in other studies. ${ }^{34,35}$

It can be seen from Table 1 and Table S3 that the (1102) planes of NZP and NZS have similar surface energies (0.69 $\mathrm{J} \mathrm{m}^{-2}$ and $0.67 \mathrm{~J} \mathrm{~m}^{-2}$, respectively), even though the latter surface contains a larger number of undercoordinated $\mathrm{Na}^{+}$ions. The similarity between the different materials suggests that the nature of the tetrahedral $\mathrm{Si}^{4+}$ or $\mathrm{P}^{5+}$ ion has less of an impact on the surface energy than the coordination of the $\mathrm{Zr}^{4+}$ ion.

Following these preliminary calculations, the influence of a surface $\mathrm{Na}_{3} \mathrm{PO}_{4}$ film on the surface energy of $\mathrm{Na}_{1+x} \mathrm{Zr}_{2} \mathrm{Si}_{x} \mathrm{P}_{3-x} \mathrm{O}_{12}$ phases could be investigated by adding a single layer of $\mathrm{Na}_{3} \mathrm{PO}_{4}$ to the low energy (1102) surfaces of NZP and NZS. A short (4 ps) ab initio molecular dynamics run at $500 \mathrm{~K}$ was used to equilibrate the surface atomic positions, followed by structural optimization. The resulting surfaces are shown in Figure 4 . For both materials, the $\mathrm{PO}_{4}$ groups in the $\mathrm{Na}_{3} \mathrm{PO}_{4}$ layer are bound to the fivefold coordinated $\mathrm{Zr}$ sites on the surfaces, resulting in a stable $\mathrm{ZrO}_{6}$ octahedron. From Table 1, it can be seen that the surface energy of the $\mathrm{Na}_{3} \mathrm{PO}_{4}$ capped NZP and NZS (11102) surfaces is lower than the corresponding bare surfaces. In other words, a $\mathrm{Na}_{3} \mathrm{PO}_{4}$ terminated NZSP surface is more thermodynamically favorable than a bare NZSP surface. The lower energy of the $\mathrm{Na}_{3} \mathrm{PO}_{4}$ capped surfaces provides a fundamental explanation for the presence of $\mathrm{Na}_{3} \mathrm{PO}_{4}$ on the surface of $\mathrm{Na}_{3.4} \mathrm{Zr}_{2} \mathrm{Si}_{2.4} \mathrm{P}_{0.6} \mathrm{O}_{12}$.

Although the current analysis of $\mathrm{Na}_{3} \mathrm{PO}_{4}$ surface layers was limited to the (1102) surface, it is likely that the presence of $\mathrm{Na}_{3} \mathrm{PO}_{4}$ will also have an impact on higher energy surfaces, particularly ones with low $\mathrm{Zr}$ coordination. A single monolayer of $\mathrm{Na}_{3} \mathrm{PO}_{4}$ was also adopted in the current study to limit the computational cost, however the exact thickness of the $\mathrm{Na}_{3} \mathrm{PO}_{4}$ layer will be the subject of future work.

\begin{tabular}{ccccc}
\hline & \multicolumn{3}{c}{ Surface Energy $\left(\mathrm{J} \mathrm{m}^{-2}\right)$} \\
\cline { 2 - 5 } Plane & \multicolumn{2}{c}{$\mathrm{NaZr}_{2} \mathrm{P}_{3} \mathrm{O}_{12}$} & \multicolumn{2}{c}{$\mathrm{Na}_{4} \mathrm{Zr}_{2} \mathrm{Si}_{3} \mathrm{O}_{12}$} \\
\cline { 2 - 5 } & Bare & $\begin{array}{c}\mathrm{Na}_{3} \mathrm{PO}_{4} \\
\text { terminated }\end{array}$ & Bare & $\begin{array}{c}\mathrm{Na}_{3} \mathrm{PO}_{4} \\
\text { terminated }\end{array}$ \\
\hline$(1 \overline{1} 02)$ & 0.69 & 0.44 & 0.67 & 0.58 \\
$(10 \overline{1} 4)$ & 0.86 & - & 0.88 & - \\
$(11 \overline{2} 0)$ & 1.15 & - & 1.26 & - \\
$(0003)$ & 0.97 & - & 0.89 & - \\
\hline \hline
\end{tabular}

Table 1. DFT computed surface energies for selected $\mathrm{NaZr}_{2} \mathrm{P}_{3} \mathrm{O}_{12}$ and $\mathrm{Na}_{4} \mathrm{Zr}_{2} \mathrm{Si}_{3} \mathrm{O}_{12}$ surfaces.

The surface energies for both stoichiometric and $\mathrm{Na}_{3} \mathrm{PO}_{4}$ capped (11̄02) surfaces are given. 

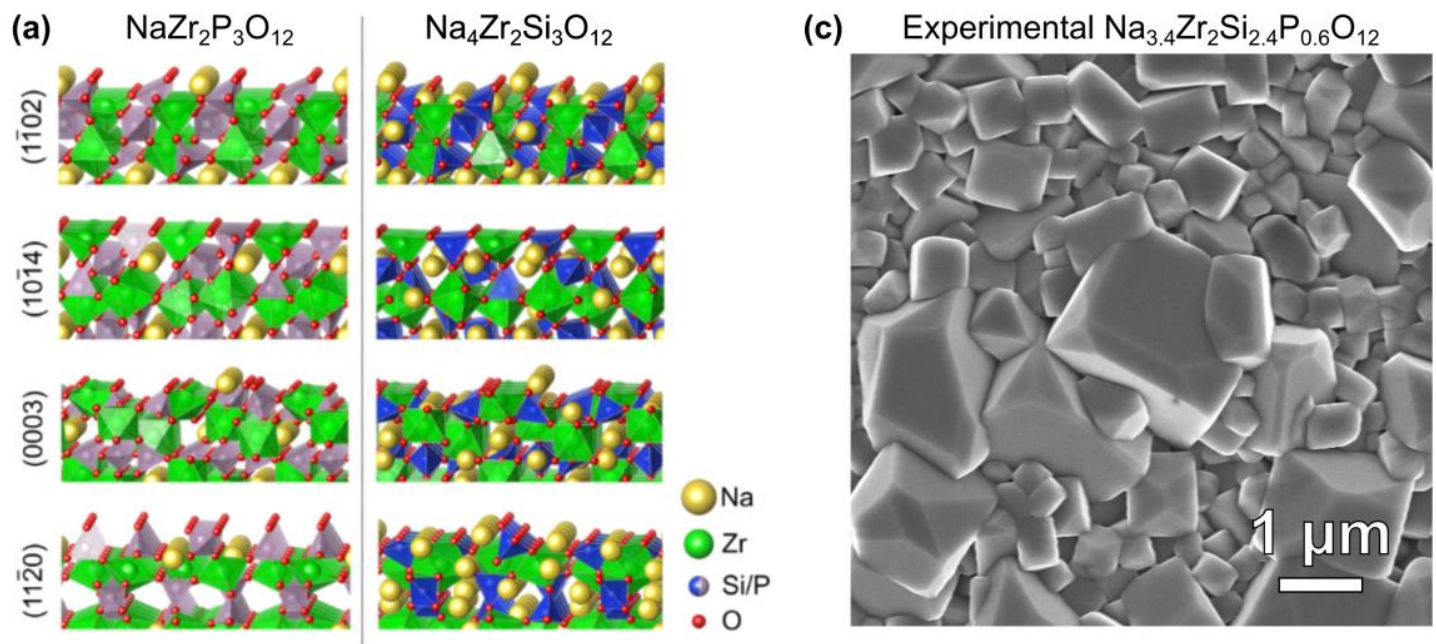

(b)
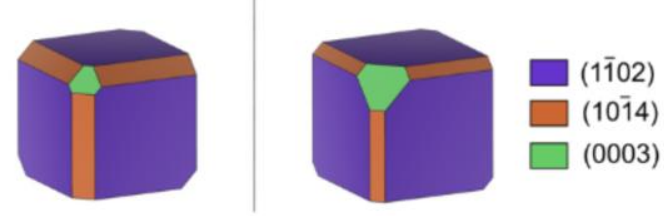

(d)

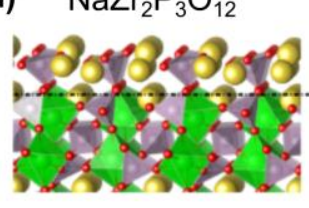

$\mathrm{Na}_{4} \mathrm{Zr}_{2} \mathrm{Si}_{3} \mathrm{O}_{12}$

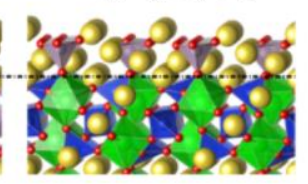

Figure 4. Surface energy DFT calculations of $\mathrm{Na}_{3} \mathrm{PO}_{4}$ terminated NZS/NZP

(A) DFT optimized surface structures and (B) Wulff energy plot for selected stoichiometric $\mathrm{NaZr}_{2} \mathrm{P}_{3} \mathrm{O}_{12}$ and $\mathrm{Na}_{4} \mathrm{Zr}_{2} \mathrm{Si}_{3} \mathrm{O}_{12}$ surfaces. (C) SEM image of $\mathrm{Na}_{3.4} \mathrm{Zr}_{2} \mathrm{Si}_{2.4} \mathrm{P}_{0.6} \mathrm{O}_{12}$ showing corresponding cube like grain morphology. (D) DFT optimized $\mathrm{NaZr}_{2} \mathrm{P}_{3} \mathrm{O}_{12}$ and $\mathrm{Na}_{4} \mathrm{Zr}_{2} \mathrm{Si}_{3} \mathrm{O}_{12}$ (11)02) surfaces capped with a monolayer of $\mathrm{Na}_{3} \mathrm{PO}_{4}$. The termination plane of the original stoichiometric (uncapped) surface is indicated with a black dashed line.

\section{Fast-charging capability of NZSP electrolytes}

Having demonstrated that the minimization of the Na|NZSP interface resistance is correlated to the in-situ formation of a thin layer on the NZSP surface, we now investigate how this chemical tuning impacts the cycling performance of NZSP electrolytes. Conventionally, the ability of SEs to withstand high current densities is studied by critical current density (CCD) measurements. Supplementary Fig. 16 shows such a CCD experiment for a symmetrical cell with a thermally activated NZSP sample subjected to a stepwise increase in current density from 0.5 to $15 \mathrm{~mA} \mathrm{~cm}{ }^{-2}$ at a constant plating/stripping capacity of $0.25 \mathrm{mAh} \mathrm{cm}{ }^{-2}$. The test was conducted at room temperature and without externally applied pressure (other than the wave spring used in the coin cell). A maximum stripping current density of $10 \mathrm{~mA} \mathrm{~cm}^{-2}$ could be reached before a soft short-circuit was observed on the next plating cycle (as the two electrodes in symmetrical cells are Na metal, the "plating" direction was arbitrarily attributed to negative current densities in Supplementary Fig.16). In contrast, when a finely polished NZSP sample was employed (as in Supplementary Fig.17 with a NZSPEtOH4000 pellet), the CCD was only $0.3 \mathrm{~mA} \mathrm{~cm}^{-2}$ before a soft short-circuit occurred. Previous studies have established that the formation of pores at the metal|SE interface under fast stripping rates are responsible for the nucleation and rapid propagation of dendrites on subsequent plating cycles. ${ }^{18,20,24}$ The formation of pores at the Na|NZSP interface is suggested by the gradual increase in cell potential over each half-cycle (more clearly observable from $4 \mathrm{~mA}$ $\mathrm{cm}^{-2}$ onwards in Supplementary Fig.16). We speculate that the large CCD obtained with thermally activated 
NZSP can be explained by the good $\mathrm{Na}$ wettability of their $\mathrm{Na}_{3} \mathrm{PO}_{4}$ surface which delays the formation of interfacial pores.

To ensure that NZSP electrolytes will operate at commercially relevant plating/stripping capacities under such high current densities, a solution to delay the apparition of interfacial pores is required. Formation cycles can be used for that purpose as illustrated in Figure 5. By gradually increasing the galvanostatic dwell duration at the same time as the current density (which we here call formation cycles), a stable plating of Na metal for 15 minutes at $10 \mathrm{~mA} \mathrm{~cm}^{-2}$ can be obtained ( $2.5 \mathrm{mAh} \mathrm{cm} \mathrm{m}^{-2}$ cycled capacity). A stripping cycle for $0.5 \mathrm{~h}$ at $10 \mathrm{~mA}$ $\mathrm{cm}^{-2}$ can even be held although resulting in a large increase in the cell resistance (up to $798 \Omega$ ) and in cell failure on the subsequent plating cycle. Considering that this performance was obtained for a planar interface, without any externally applied pressure, and at room temperature, these results predict the high potential of NZSP as a SE for high power application. Strategies enabling the suppression of interfacial pores and extended cycling of NZSP cells will be investigated as part of future work.

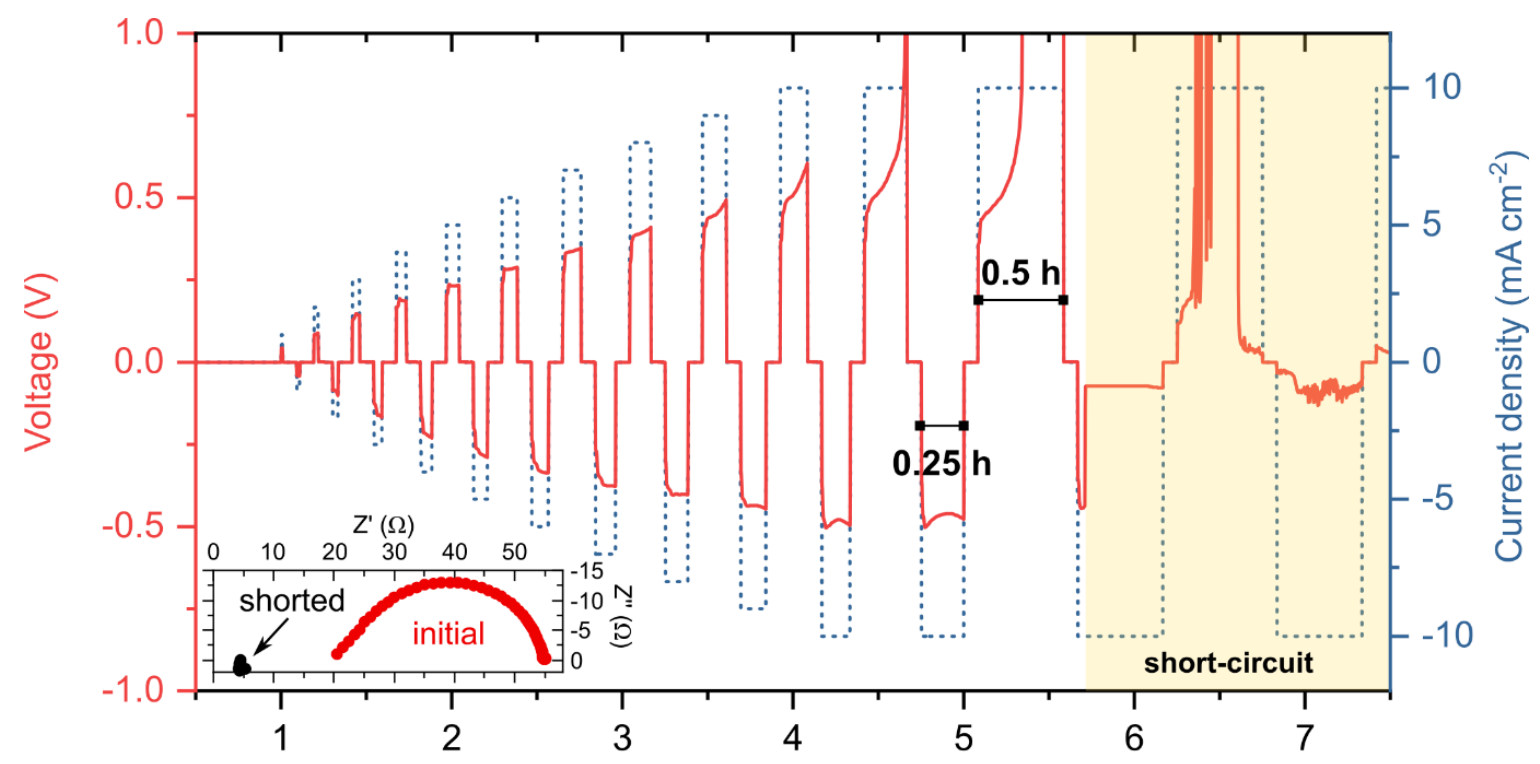

Time (h)

Figure 5. Fast-charging capability of NZSP electrolytes

Voltage profile of a Na|NZSP as sintered| $\mathrm{Na}$ cell subject to a simultaneous increase in current densities (from 1 to $10 \mathrm{~mA} \mathrm{~cm}^{-}$ $\left.{ }^{2}\right)$ together with an increase in the galvanostatic dwell durations (from $30 \mathrm{sec}$ to $0.5 \mathrm{~h}$ ). Impedance spectra before cycling and after the short-circuit are shown in inset. NZSP pellet thickness: $1.70 \mathrm{~mm}$; Na metal anodes diameter: $8.5 \mathrm{~mm}$; electrode capacities: 11 mAh (equivalent thickness: $182 \mu \mathrm{m}$ ); measured at room temperature.

\section{DISCUSSION}

By design, alkali metal solid-state batteries approach the question of metal plating with a radically different perspective than liquid electrolyte batteries with intercalation anodes. Whilst for the latter, metal plating is a safety hazard putting a cap on their charging rate, for solid-state batteries, metal plating is the fundamental mechanism by which charging occurs. Therefore, the anode|SE interface in SSBs is in theory not rate limiting. 
Yet, a crucial requirement to ensure that SEs can withstand such high current densities is that the plating occurs homogeneously across the interface and not at localized constriction points.

The complex nature of an experimental alkali metal|SE interface creates a challenge for separating the relative contributions of distinct ionically blocking processes. For such interfaces, the interface resistance and charge transfer resistance cannot be confused: while the interface resistance corresponds to the apparent sum of all interface processes with a resistive contribution, the charge transfer resistance only accounts for the resistance associated with the $\mathrm{Na}^{0}{ }_{\text {anode }} \leftrightarrow \mathrm{Na}^{+}$SE $+\mathrm{e}^{-}$anode conversion across a perfect interface. Hence, charge transfer is only one contribution to the overall interface resistance. In a recent study, Krauskopf et al. were able to carefully produce a pristine Li|LLZO interface, virtually free from any contamination: an extremely low interface resistance of $0.1 \Omega \mathrm{cm}^{2}$ at room temperature was measured and attributed to charge transfer across the Li|LLZO interface. ${ }^{28}$ With this study, Krauskopf et al. proved, at least for the Li|LLZO interface, that charge transfer is a marginal contribution to the interface resistance and that all resistive contributions above $0.1 \Omega$ $\mathrm{cm}^{2}$ should be attributed to other ionically blocking processes.

Pragmatically, contaminant-free and perfectly wetting interfaces are difficult to prepare and manipulate. Therefore, under more conventional experimental conditions, the akali metal|SE interface resistance is predominantly affected by (i) pores between the metal anode and SE resulting in improper contact, ${ }^{36}$ (ii) adventitious contaminants sandwiched between the anode and the SE (layers formed on the surface of the SE or metal anode by reaction with a gas environment prior to cell assembly) ${ }^{19,29-31,37}$ or (iii) solid electrolyte interphases (SEI, degradation products formed at the interface resulting from the thermodynamic instability of the SE against the metal anode). The existence of a rapidly forming SEI at the Na|NZSP interface was recently suggested by several studies. ${ }^{38-41}$

With the aim to isolate the contribution of charge transfer from other resistive contributions to the interface resistance, in this study, a uniaxial load of $500 \mathrm{~N}$ was systematically applied during cell assembly (not during electrochemical testing) to guarantee an intimate contact between the SE and $\mathrm{Na}$ metal and thus minimize constriction resistance effects. The contribution of an SEI to the measured interfacial resistance was mitigated by measuring the cells quickly after cell assembly (typically 30 minutes after cell assembly). Besides, by measuring the impedance of a symmetrical cell 4 month after its assembly, we demonstrate that the contribution of the SEI to the interface resistance can be limited for NZSP as sintered to $5 \Omega \mathrm{cm}^{2}$ (Supplementary Fig.18).

The normalized interfacial resistances from all symmetrical cells tested in this study are presented in Figure 6. This figure illustrates the range of interfacial resistances which can be obtained for the same alkali metal | SE system when the processing of the SE surface is changed.

Contaminants on the surface of SEs are responsible for large alkali metal|SE interfacial resistances in a variety of systems. Already during the development of the Na-S battery, contaminants on the surface of Na- $\beta$ "'-alumina tubes were linked to larger interface resistances and an increased risk of dendrite penetration. ${ }^{42} \mathrm{~A}$ more recent study by Bay et al. demonstrated the detrimental impact of surface hydroxides and carbonates on the $\mathrm{Na} \mid \mathrm{Na}$ - 
$\beta^{\prime \prime}$-alumina interface resistance. ${ }^{29}$ The Li|LLZO interface is another good example where contaminants (e.g. $\mathrm{Li}_{2} \mathrm{CO}_{3}$ and $\mathrm{LiOH}$ ) on the surface of LLZO pellets lead to large interface resistances. ${ }^{19,30,31,43}$ In line with the conclusions from other groups, we also observe that adventitious carbon species negatively affect the Na|NZSP interfacial resistance. NZSP samples with more carbon contaminants on their surface (such as NZSPdry4000 and NZSPEtoH4000, see Supplementary Fig.7 and Supplementary Fig.8) can be linked to large $\mathrm{Na} \mid \mathrm{NZSP}$ interface resistances.

Nonetheless, the negligible Na|NZSP interface resistance obtained with thermally activated NZSP samples should not be only attributed to a thermally induced surface cleaning mechanism. The interface resistance of cells made with NZSPEtOH500 pellets, whose surface showed similar level of adventitious C contamination (see Figure 2), should otherwise also be negligible. Instead, we attribute the low Na|NZSP interface resistance to the good Na wettability of thermally activated NZSP. The surface analyses presented in this study demonstrate that thermal activation induces a thermodynamic reconstruction of NZSP surfaces. Whichever polishing conditions are employed, a post-polish annealing always leads to the stabilization of a thin surface $\mathrm{Na}_{3} \mathrm{PO}_{4}$ film. The existence of self-formed surface amorphous films (or complexions) on various ceramics has recently gained more scientific attention ${ }^{14,44-46}$ and our results suggest that NaSICON ceramics may also form such thermodynamically favorable surfaces.

The presence of this surface layer was observed on all thermally activated samples presented in this study and was correlated to negligible interface resistances (down to $0.1 \Omega \mathrm{cm}^{2}$ ). The high sodiophilicity of this surface layer is suggested by the ability of thermally activated NZSP to be stably cycled at $10 \mathrm{~mA} \mathrm{~cm}^{-2}$ (which corresponds to $24 \mathrm{~nm}$ of $\mathrm{Na}$ metal being consumed from the stripping electrode every second) for extended periods of time. Finally, although more experiments would be required to confirm this hypothesis, the $\mathrm{Na}_{3} \mathrm{PO}_{4}$ layer present on thermally activated NaSICON surfaces could act as a buffer layer preventing the formation of a resistive SEI due to its stability against Na metal. ${ }^{47}$ This would explain why, unlike other recent studies, ${ }^{41,48}$ we can report an interface resistance increase after 4 months of only $5 \Omega \mathrm{cm}^{2}$ (Supplementary Fig.18). 


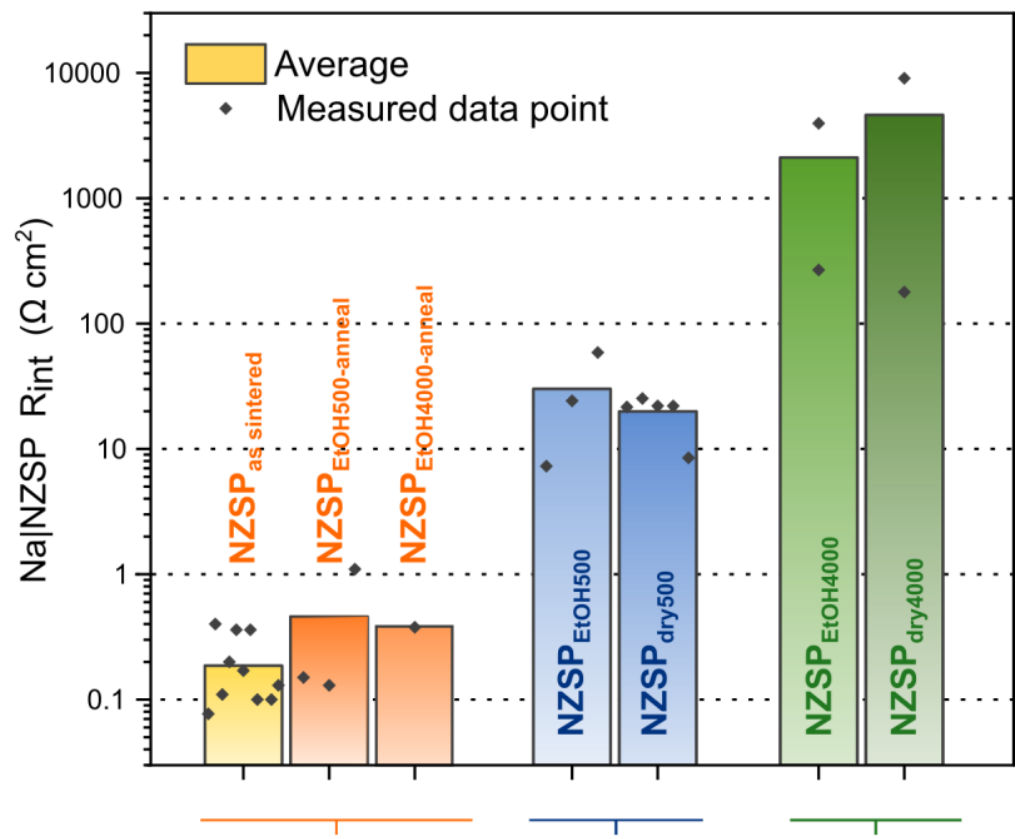

Thermally activated Rough polish Fine polish

Figure 6. Interface resistances of $\mathrm{Na|NZSP|Na} \mathrm{symmetrical} \mathrm{cells}$

Bar chart of mean interface resistance values obtained with $\mathrm{Na|NZSP|Na}$ symmetrical cells

\section{CONCLUSION}

Enabling fast charging SSBs requires to optimize the interface between alkali metal anodes and SEs. In this study, we introduced a simple procedure to improve the affinity of NZSP towards Na metal. We demonstrated that thermally activating NZSP samples (either by sintering or annealing pellets after polishing) results in the in-situ formation of a thermodynamically favorable $\mathrm{Na}_{3} \mathrm{PO}_{4}$ surface layer. The $\mathrm{Na}$ |NZSP interface resistance of cells employing thermally activated NZSP pellets is consistently below $1 \Omega \mathrm{cm}^{2}$. The ability of thermally activated NZSP samples to withstand high current densities and homogeneously plate $\mathrm{Na}$ metal was demonstrated by their critical current density of $10 \mathrm{~mA} \mathrm{~cm}^{-2}\left(5 \mathrm{mAh} \mathrm{cm}^{-2}\right.$ cycled capacity).

Overall, a robust procedure to optimize the Na|NZSP interface is presented. The excellent performance of assintered NZSP samples is very promising for industrial prospects because no additional processing steps are required to obtain stable anodic half-cells.

\section{METHODS}

\section{NZSP synthesis}

$\mathrm{Na}_{3.4} \mathrm{Zr}_{2} \mathrm{Si}_{2.4} \mathrm{P}_{0.6} \mathrm{O}_{12}$ was synthesized following a solution-assisted solid-state synthesis. ${ }^{13} \mathrm{NaNO}_{3}$ (Sigma Aldrich, 99\%), $\mathrm{ZrO}\left(\mathrm{NO}_{3}\right)_{2}$ (Sigma Aldrich, 99\%), $\mathrm{Si}\left(\mathrm{OC}_{2} \mathrm{H}_{5}\right)_{4}$ (Sigma Aldrich, 99\%) and $\mathrm{NH}_{4} \mathrm{H}_{2} \mathrm{PO}_{4}$ (Sigma Aldrich, 99.5\%) were used as starting materials. To act as a sinter aid, an off-stoichiometric amount of $\mathrm{NaNO}_{3}$ and $\mathrm{NH}_{4} \mathrm{H}_{2} \mathrm{PO}_{4}$ was added in the ratio $\mathrm{Na}_{3.4} \mathrm{Zr}_{2} \mathrm{Si}_{2.4} \mathrm{P}_{0.6} \mathrm{O}_{12}: 1.5 \% \mathrm{Na}_{3} \mathrm{PO}_{4}$. 
Stoichiometric amounts of $\mathrm{NaNO}_{3}$ and $\mathrm{ZrO}\left(\mathrm{NO}_{3}\right)_{2}$ were first dissolved in deionized water. Concentrated $\mathrm{HNO}_{3}$ was added while stirring to help with the dissolution of precursors. The volume of $\mathrm{HNO}_{3}$ represented $5 \%$ of the total final volume. $\mathrm{Si}\left(\mathrm{OC}_{2} \mathrm{H}_{5}\right)_{4}$ was added in stoichiometric proportions and the solution was stirred until complete dissolution of all precursors was observed. Once fully dissolved, the last precursor, $\left(\mathrm{NH}_{4}\right) \mathrm{H}_{2} \mathrm{PO}_{4}$, was added to the solution. The addition of $\left(\mathrm{NH}_{4}\right) \mathrm{H}_{2} \mathrm{PO}_{4}$ led to the formation of white precipitates. ${ }^{13}$ The solution was then homogenized by stirring and dried in a vacuum oven at $80^{\circ} \mathrm{C}$ overnight. The dried gel was decomposed at $800{ }^{\circ} \mathrm{C}$ for $3 \mathrm{~h}$ with a heating and cooling rate of $180^{\circ} \mathrm{C} \mathrm{h}^{-1}$. The obtained white powder was ground in an agate mortar and then ball-milled on a roller bench for $72 \mathrm{~h}$ in ethanol with zirconia balls. Pellet samples were pressed using a $13 \mathrm{~mm}$ diameter die under a load of $1.5 \mathrm{~T}$. Pellets were sintered in $\mathrm{Pt}$ crucibles or $\mathrm{Al}_{2} \mathrm{O}_{3}$ crucibles lined with $\mathrm{Pt}$ foil $\left(1285^{\circ} \mathrm{C}, 6 \mathrm{~h}, 180^{\circ} \mathrm{C} \mathrm{h}^{-1}\right.$ heating and cooling rates).

The relative density of NZSP pellets used in this study was estimated geometrically to be $96 \pm 2 \%$.

\section{NZSP surface processing and nomenclature}

Pellets were polished with either SiC (Struers, grit sizes: 500, 1200, 2400, 4000). For some samples, ethanol (VWR ethanol absolute $>99.8 \%$ ) was used as a lubricating solvent. To remove surface residues after polishing, pellets were either sonicated in ethanol for 1 minute or dusted with compressed air canisters for dry polished samples.

Selected samples were annealed after polishing in a muffle furnace at $900{ }^{\circ} \mathrm{C}$, for $0.5 \mathrm{~h}$ with a $900{ }^{\circ} \mathrm{C} \mathrm{h}-1$ heating and cooling rate.

The following nomenclature is used to distinguish the various NZSP samples in the manuscript (see Table 2): ethanol is abbreviated as $\mathrm{EtOH}$; the term "dry" refers to a polishing condition without solvent; a post-polishing annealing step is indicated by the term "anneal" in the subscript. For example, NZSPEtoH500-anneal is an NZSP pellet which was polished with SiC paper of grit size 500, using ethanol as solvent, followed by an annealing step at $900^{\circ} \mathrm{C}$. NZSP as sintered refers to samples which were neither polished nor annealed. Finally, NZSP xsection refers to a sample which was snapped in half to analyze the chemical composition of the center of a pellet.

\begin{tabular}{|c|c|c|c|}
\hline \multicolumn{2}{|c|}{ Polishing } & \multirow{2}{*}{ Annealing } & \multirow{2}{*}{ Sample's name } \\
\hline Solvent & SiC grit size & & \\
\hline- & - & - & $\mathrm{NZSP}_{\text {as sintered }}$ \\
\hline Ethanol & 500 & - & NZSP $P_{E \dagger O H 500}$ \\
\hline Ethanol & 500 & $900{ }^{\circ} \mathrm{C}$ for $0.5 \mathrm{~h}$ & $\mathrm{NZSP}_{\text {EtOH} 500-\text { anneal }}$ \\
\hline Ethanol & 4000 & - & NZSP EtOH 4000 \\
\hline Ethanol & 4000 & $900{ }^{\circ} \mathrm{C}$ for $0.5 \mathrm{~h}$ & NZSPEtOH4000- \\
\hline $\begin{array}{l}\text { Dry (no } \\
\text { solvent) }\end{array}$ & 500 & - & NZSP $_{\text {dry500 }}$ \\
\hline $\begin{array}{l}\text { Dry (no } \\
\text { solvent) }\end{array}$ & 4000 & - & $\mathrm{NZSP}_{\text {dry } 4000}$ \\
\hline
\end{tabular}

Table 2. NZSP samples nomenclature

\section{NZSP structure and microstructure characterization}

The crystal structure and purity of NZSP samples were tested by XRD on a PANalytical MPD powder diffractometer with monochromatic $\mathrm{Cu} \mathrm{K}_{\alpha 1}$ radiation $(\lambda=1.540598 \AA)$. Patterns were collected in a $2 \theta$ range between 10 and $80^{\circ}$ with a step size of $0.033^{\circ}$ and a $3 \mathrm{~s}$ collection time at each step. The crystalline structure of NZSP samples was analyzed by X-ray diffraction (XRD). X-ray diffractograms are presented in Supplementary Fig.1. The reader is referred to a previous publication for details on the NZSP crystal structure. ${ }^{13}$

SEM images were obtained using the in-lens detector of a Zeiss Leo Gemini 1525 at a working distance of 7 $\mathrm{mm}$ with an electron beam accelerating voltage of $5 \mathrm{kV}$ and a $30 \mu \mathrm{m}$ aperture. EDX spectra were measured with an Oxford Instrument $\mathrm{x}$-act detector using the same beam excitation. A low accelerating voltage was 
chosen to ensure a reduced interaction volume and higher surface sensitivity. The low X-ray production rate was compensated by longer integration times.

\section{Cell assembly}

$\mathrm{Na}$ metal films were freshly prepared for each assembled cell in an Ar-filled glovebox. A clean piece of $\mathrm{Na}$ was cut from a rod (Na sticks, covered in film of protective hydrocarbon oil, 99\%, Alfa Aesar), then pressed flat in a LDPE plastic bag to a thickness of 100-300 $\mu \mathrm{m}$. Circular electrodes were then punched from this foil. Their surface was mechanically cleaned using the blade of a scalpel. The Na metal electrodes were then placed on both sides of a NZSP pellet and the Na|NZSP|Na stack was pressed with a uniaxial pressure of around 10 $\mathrm{MPa}$. The applied pressure was measured using a KMM50-10kN force sensor (inelta Sensorsysteme $\mathrm{GmbH}$ ). The diameter of the Na metal electrodes was measured after the cell was pressed. For electrochemical tests requiring instruments outside the glovebox, the symmetrical cells were placed in stainless steel coin cells.

\section{Electrochemical measurements}

Impedance spectra were measured on a Solartron 1260 and a Biologic VMP-300 frequency response analyzers (FRA) with an excitation amplitude of $\mathrm{V}_{\mathrm{A} . \mathrm{C} .}=50 \mathrm{mV}$. For the Solartron measurements, data points were collected in the frequency range of $13 \mathrm{MHz}$ to $5 \mathrm{~Hz}(7 \mathrm{MHz}$ to $1 \mathrm{~Hz}$ for the Biologic) with a logarithmic sampling at 20 points/decade. To minimize the signal to noise ratio, the frequency range was split into three sub-ranges $(13 \mathrm{MHz}-500 \mathrm{kHz}, 500 \mathrm{kHz}-500 \mathrm{~Hz}, 500 \mathrm{~Hz}-5 \mathrm{~Hz}$ ) with varying integration times and delays (2 sec integration and 10 cycles delay; $1 \mathrm{sec}$ integration and 5 cycles delay; 30 cycles integration and 3 cycles delay, respectively). The temperature was maintained at $25^{\circ} \mathrm{C}$ using a Linkam HFS600 E-P temperature control stage. Results were fitted using RelaxIS3 (Rhd instruments). The reported capacitance values were calculated using the Brug formula:

$$
C=\frac{(Q * R)^{1 / n}}{R}
$$

Where $\mathrm{Q}$ and $\mathrm{n}$ are the parameters of the Constant Phase Elements used in the equivalent circuit models. As impedance spectra were measured on two different FRAs, a difference in the systems' ohmic resistances was noticed. For samples measured on the instrument with higher ohmic resistance, the spectra were shifted so that the high frequency intercept with the real axis corresponds to a bulk conductivity of $15 \mathrm{mS} \mathrm{cm}^{-1}$ (as previously measured). ${ }^{13}$

The CCD of Na|NZSP|Na symmetric cells was tested using a Scribner 580 battery cycler. Cells were typically cycled between $0.5 \mathrm{~mA} \mathrm{~cm}^{-2}$ and $15 \mathrm{~mA} \mathrm{~cm}^{-2}$ until signs of a short-circuit were observed. Depending on the measurement, either the cycled capacity or the duration of the galvanostatic dwell were kept constant. Formation cycles consists in a simultaneous stepwise increase of the cycling current density (from 1 to $10 \mathrm{~mA}$ $\mathrm{cm}^{-2}$ ) and dwell time (from $30 \mathrm{sec}$ to $0.5 \mathrm{~h}$ ).

\section{X-ray photoelectron spectroscopy (XPS)}

XPS spectra were collected at room temperature on a Thermo Fisher Scientific XPS system with a monochromated Al Ka source $(1486.6 \mathrm{eV})$ operating at a $2 \times 10^{-9} \mathrm{mbar}$ base pressure. The X-ray source was operated at $6 \mathrm{~mA}$ emission current and $12 \mathrm{kV}$ anode bias providing a $400 \mu \mathrm{m}^{2} \mathrm{X}$-ray spot size. Core level spectra were measured using a pass energy of $20 \mathrm{eV}$, at a resolution of $0.1 \mathrm{eV}$ and an integration time of 50 $\mathrm{ms} /$ point. Averages over a number of scans varying from 30 for intense signals (Na1s for instance) to 80 for lower intensity signals (P2p) were taken. Sample charging was neutralized using a low energy electron flood gun $(0.2 \mathrm{~V}, 100 \mu \mathrm{A})$. Results were fitted using the Avantage software (Thermo Fisher Scientific). The background was fitted using a modified Shirley type function which includes a condition of non-intersection between the background and the data points. The Zr3d5 peak position was used as a reference for charge compensation (Zr3d5 fixed at $182.40 \mathrm{eV}$ ) and intensity normalization.

\section{Low energy ion scattering (LEIS)}

The LEIS analysis was carried out in an Ion-ToF Qtac100 instrument by ION-TOF GmbH. The Qtac energy analyzer is extremely surface sensitive providing quantitative elemental characterization of the first atomic layer of the sample. The base pressure in the analysis chamber was $\sim 9 \times 10^{-10} \mathrm{mbar}$. During the experiment, the chamber pressure increases to $10^{-8} \mathrm{mbar}$ because of the noble gas flux from the ion source. For the measurements, a $3 \mathrm{keV}{ }^{4} \mathrm{He}^{+}$ion beam was used. $\mathrm{A} \mathrm{He}^{+}$ion source was chosen because of its high sensitivity to low mass atoms. The primary ion beam was aligned perpendicular to the samples' surface and the scattered ions were detected at an angle of $145^{\circ}$. The primary ion beam was scanned on a sample surface area of 500 
$\mu \mathrm{m} \times 500 \mu \mathrm{m}$. To prevent charging effects due to the insulating nature of NaSICON samples, a low-energy electron flood gun $(10 \mathrm{eV})$ was used.

The energy of each peak position can be calculated according to the laws of conservation of energy and momentum. ${ }^{49}$ The primary ions that overcome the first atomic layer are mostly neutralized. The ones that are both scattered from deeper sample layers and re-ionized by the interaction with the oxide surface lead to an in-depth signal, which appears in the spectra as a left background shoulder with respect to the elastic atomic peak position. More details about the technique can be found elsewhere. ${ }^{50}$

With this study, a preferential sputtering of $\mathrm{Na}$ atoms (even at a low sputtering energy of $0.5 \mathrm{keV}$ ) was noticed. As the ion dose increases the sodium signal decreases. Sodium depletion from $\mathrm{Ar}^{+}$-bombarded silicate glass surfaces has already been reported. ${ }^{51}$ For this reason, the area of the Na peak was not used in the normalized results. More specifically, the normalized results were obtained by dividing the area of each peak (obtained by curve fitting) to the total area of all detectable peaks.

\section{First principles calculations}

Density functional theory (DFT) calculations of end member $\mathrm{NaZr}_{2} \mathrm{P}_{3} \mathrm{O}_{12}$ (NZP) and $\mathrm{Na}_{4} \mathrm{Zr}_{2} \mathrm{Si}_{3} \mathrm{O}_{12}$ (NZS) NaSICON compositions were performed with the Vienna ab initio simulation package (VASP), using the Perdew-Burke-Ernzerhof (PBE) exchange-correlation functional. ${ }^{52,53}$ Projector-augmented wave (PAW) pseudopotentials were used for all species, with a plane wave cut off of $500 \mathrm{eV} .{ }^{54}$ To minimize the computational cost, the softest PAW pseudopotentials from each element were used from the VASP 5.4.4 distribution, which are labelled $\mathrm{Na}, \mathrm{Zr}$ _sv, P, Si and O_s. Tests using pseudopotentials containing additional valence electrons did not result in qualitative differences to the surface energies or structures. An energy convergence of $1 \times 10^{-6} \mathrm{eV}$ or better was used for all calculations and spin polarization was included unless otherwise stated. A gamma-centered k-point mesh with mesh density of $20 \AA$ or better was used to sample the Brillouin zone, unless otherwise stated. The primitive, bulk unit cell structures of NZP and NZS $(R \overline{3} \mathrm{~m})$ were fully optimized without symmetry constraints until the force on any atom fell below $0.01 \mathrm{eV} / \AA$.

The reader is referred to the SI for more information about surface creation and surface energy calculations.

\section{SUPPLEMENTARY INFORMATION}

Document S1. Supplementary Methods, Supplementary Note 1, Supplementary Fig.1-18, and Supplementary Tables 1-3

\section{REFERENCES}

1. Albertus, P., Babinec, S., Litzelman, S. \& Newman, A. Status and challenges in enabling the lithium metal electrode for highenergy and low-cost rechargeable batteries. Nat. Energy 3, 1621 (2018).

2. Grey, C. P. \& Tarascon, J. M. Sustainability and in situ monitoring in battery development. Nat. Mater. 16, 45-56 (2016).

3. Tarascon, J.-M. \& Armand, M. Issues and challenges facing rechargeable lithium batteries. Nature 414, 359-367 (2001).

4. Bieker, G., Winter, M. \& Bieker, P. Electrochemical in situ investigations of SEI and dendrite formation on the lithium metal anode. Phys. Chem. Chem. Phys. 17, 8670-8679 (2015).

5. Banza Lubaba Nkulu, C. et al. Sustainability of artisanal mining of cobalt in DR Congo. Nat. Sustain. 1, 495-504 (2018).

6. Frankel, T. \& Whoriskey, P. Indigenous people are left poor as tech world takes lithium from under their feet. The Washington Post (2016).

7. Whoriskey, P. China pollution caused by graphite mining for smartphone battery. The Washington Post (2016).

8. Frankel, T. Cobalt mining for lithium ion batteries has a high 
human cost. The Washington Post (2016).

9. Larcher, D. \& Tarascon, J.-M. Towards greener and more sustainable batteries for electrical energy storage. Nat. Chem. 7, 19-29 (2014).

10. Ma, Q. \& Tietz, F. Solid-State Electrolyte Materials for Sodium Batteries: Towards Practical Applications. ChemElectroChem 7, 2693-2713 (2020).

11. Goodenough, J. B., Hong, H. Y.-P. \& Kafalas, J. A. Fast $\mathrm{Na}+$ ion transport in skeleton structures. Mat. Res. Bull 11, 203-220 (1976).

12. Hong, H. .-P. Crystal structures and crystal chemistry in the system Na1+xZr2SixP3-xO12. Mater. Res. Bull. 11, 173-182 (1976).

13. Ma, Q. et al. Room temperature demonstration of a sodium superionic conductor with grain conductivity in excess of $0.01 \mathrm{~S}$ $\mathrm{cm}-1$ and its primary applications in symmetric battery cells. $J$. Mater. Chem. A 7, 7766-7776 (2019).

14. Luo, J. Let thermodynamics do the interfacial engineering of batteries and solid electrolytes. Energy Storage Mater. 21, 5060 (2019).

15. Samiee, M. et al. Divalent-doped Na3Zr2Si2PO12 natrium superionic conductor: Improving the ionic conductivity via simultaneously optimizing the phase and chemistry of the primary and secondary phases. J. Power Sources 347, 229-237 (2017).

16. Okubo, K. et al. A dense NASICON sheet prepared by tapecasting and low temperature sintering. Electrochim. Acta 278, 176-181 (2018).

17. Narayanan, S., Reid, S., Butler, S. \& Thangadurai, V. Sintering temperature, excess sodium, and phosphorous dependencies on morphology and ionic conductivity of NASICON Na3Zr2Si2PO12. Solid State lonics 331, 22-29 (2019).

18. Krauskopf, T., Richter, F. H., Zeier, W. G. \& Janek, J. Physicochemical Concepts of the Lithium Metal Anode in SolidState Batteries. Chemical Reviews 120, 7745-7794 (2020).

19. Sharafi, A. et al. Surface Chemistry Mechanism of Ultra-Low Interfacial Resistance in the Solid-State Electrolyte Li7La3Zr2O12. Chem. Mater. 29, 7961-7968 (2017).

20. Wang, M. J., Choudhury, R. \& Sakamoto, J. Characterizing the Li-Solid-Electrolyte Interface Dynamics as a Function of Stack Pressure and Current Density. Joule 3, 2165-2178 (2019).

21. Pesci, F. M. et al. Elucidating the role of dopants in the critical current density for dendrite formation in garnet electrolytes. $J$. Mater. Chem. A 6, 19817-19827 (2018).

22. Wang, D., Zhu, C., Fu, Y., Sun, X. \& Yang, Y. Interfaces in Garnet-Based All-Solid-State Lithium Batteries. Adv. Energy Mater. 10, 2001318 (2020).

23. Krauskopf, T., Hartmann, H., Zeier, W. G. \& Janek, J. Toward a Fundamental Understanding of the Lithium Metal Anode in SolidState Batteries-An Electrochemo-Mechanical Study on the Garnet-Type Solid Electrolyte $\mathrm{Li}_{6.25} \mathrm{Al}_{0.25} \mathrm{La}_{3} \mathrm{Zr}_{2} \mathrm{O}_{12}$. ACS Appl. Mater. Interfaces acsami.9b02537 (2019). doi:10.1021/acsami.9b02537

24. Kasemchainan, J. et al. Critical stripping current leads to dendrite formation on plating in lithium anode solid electrolyte 
cells. Nat. Mater. 1-7 (2019). doi:10.1038/s41563-019-0438-9

25. Spencer Jolly, D. et al. Sodium/Na $\beta^{\prime \prime}$ Alumina Interface: Effect of Pressure on Voids. ACS Appl. Mater. Interfaces 12, 678-685 (2020).

26. Hitz, G. T. et al. High-rate lithium cycling in a scalable trilayer Ligarnet-electrolyte architecture. Mater. Today 22, 50-57 (2019).

27. Ma, Q. et al. Scandium-Substituted Na 3 Zr 2 ( $\mathrm{SiO} 4$ ) 2 (PO 4 ) Prepared by a Solution- Assisted Solid-State Reaction Method as Sodium-Ion Conductors. Chem. Mater. 28, 4821-4828 (2016).

28. Krauskopf, T. et al. The Fast Charge Transfer Kinetics of the Lithium Metal Anode on the Garnet-Type Solid Electrolyte Li 6.25 Al $0.25 \mathrm{La}_{3} \mathrm{Zr} 2 \mathrm{O}$ 12. Adv. Energy Mater. 2000945 (2020). doi:10.1002/aenm.202000945

29. Bay, M. et al. Sodium Plating from Na- $\beta$ "-Alumina Ceramics at Room Temperature, Paving the Way for Fast-Charging All-SolidState Batteries. Adv. Energy Mater. 1902899 (2019). doi:10.1002/aenm.201902899

30. Huo, H. et al. Li2CO3 : A Critical Issue for Developing Solid Garnet Batteries. ACS Energy Lett. 5, 252-262 (2020).

31. Meng, J., Zhang, Y., Zhou, X., Lei, M. \& Li, C. Li2CO3-affiliative mechanism for air-accessible interface engineering of garnet electrolyte via facile liquid metal painting. Nat. Commun. 11, 3716 (2020).

32. Kramer, D. \& Ceder, G. Tailoring the morphology of LiCoO2: A first principles study. Chem. Mater. 21, 3799-3809 (2009).

33. Wang, L., Zhou, F., Meng, Y. S. \& Ceder, G. First-principles study of surface properties of LiFeP O4: Surface energy, structure, Wulff shape, and surface redox potential. Phys. Rev. $B$ - Condens. Matter Mater. Phys. 76, 165435 (2007).

34. Naqash, S., Ma, Q., Tietz, F. \& Guillon, O. Na3Zr2(SiO4)2(PO4) prepared by a solution-assisted solid state reaction. Solid State lonics 302, 83-91 (2017).

35. Jalalian-Khakshour, A. et al. Solid-state synthesis of NASICON (Na3Zr2Si2PO12) using nanoparticle precursors for optimisation of ionic conductivity. J. Mater. Sci. 55, 2291-2302 (2020).

36. Krauskopf, T., Hartmann, H., Zeier, W. G. \& Janek, J. Toward a Fundamental Understanding of the Lithium Metal Anode in SolidState Batteries - An Electrochemo-Mechanical Study on the Garnet-Type Solid Electrolyte Li6.25AI0.25La3Zr2O12. ACS Appl. Mater. Interfaces 11, 14463-14477 (2019).

37. Etxebarria, A. et al. Work Function Evolution in Li Anode Processing. Adv. Energy Mater. 2000520 (2020). doi:10.1002/aenm.202000520

38. Yang, J. et al. Guided-formation of a favorable interface for stabilizing Na metal solid-state batteries. J. Mater. Chem. A 8, 7828-7835 (2020).

39. Gao, Z. et al. Stabilizing $\mathrm{Na} 3 \mathrm{Zr} 2 \mathrm{Si} 2 \mathrm{PO} 12$ /Na Interfacial Performance by Introducing a Clean and Na-Deficient Surface . Chem. Mater. 32, 3970-3979 (2020).

40. Gao, H., Xin, S., Xue, L. \& Goodenough, J. B. Stabilizing a HighEnergy-Density Rechargeable Sodium Battery with a Solid Electrolyte. Chem 4, 833-844 (2018).

41. Wang, S., Xu, H., Li, W., Dolocan, A. \& Manthiram, A. Interfacial Chemistry in Solid-State Batteries: Formation of Interphase and 
Its Consequences. J. Am. Chem. Soc 140, 250-257 (2018).

42. Yang, Z. et al. Electrochemical energy storage for green grid. Chemical Reviews 111, 3577-3613 (2011).

43. Cheng, L. et al. The origin of high electrolyte-electrode interfacial resistances in lithium cells containing garnet type solid electrolytes. Phys. Chem. Chem. Phys. 16, 18294-18300 (2014).

44. Kaplan, W. D., Chatain, D., Wynblatt, P. \& Carter, W. C. A review of wetting versus adsorption, complexions, and related phenomena: The rosetta stone of wetting. Journal of Materials Science 48, 5681-5717 (2013).

45. Naqash, S., Sebold, D., Tietz, F. \& Guillon, O. Microstructure-

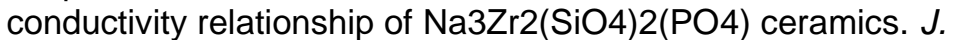
Am. Ceram. Soc. 102, 1057-1070 (2019).

46. Luo, J. Interfacial engineering of solid electrolytes. J. Mater. 1, 22-32 (2015).

47. Lacivita, V., Wang, Y., Bo, S. H. \& Ceder, G. Ab initio investigation of the stability of electrolyte/electrode interfaces in all-solid-state Na batteries. J. Mater. Chem. A 7, 8144-8155 (2019).

48. Zhang, Z. et al. Na3Zr2Si2PO12: A Stable $\mathrm{Na}+$-lon Solid Electrolyte for Solid-State Batteries. ACS Appl. Energy Mater. 19, 39 (2020).

49. Niehus, H., Heiland, W. \& Taglauer, E. Low-energy ion scattering at surfaces. Surf. Sci. Rep. 17, 213-303 (1993).

50. Brongersma, H. H., Draxler, M., de Ridder, M. \& Bauer, P. Surface composition analysis by low-energy ion scattering. Surface Science Reports 62, 63-109 (2007).

51. Smets, B. M. J. \& Lommen, T. P. A. Ion Beam Effects on Glass Surfaces. J. Am. Ceram. Soc. 65, c80-c81 (1982).

52. Kresse, G. \& Furthmüller, J. Efficient iterative schemes for $a b$ initio total-energy calculations using a plane-wave basis set. Phys. Rev. B - Condens. Matter Mater. Phys. 54, 11169-11186 (1996).

53. Perdew, J. P., Burke, K. \& Ernzerhof, M. Generalized gradient approximation made simple. Phys. Rev. Lett. 77, 3865-3868 (1996).

54. Blöchl, P. E. Projector augmented-wave method. Phys. Rev. B 50, 17953-17979 (1994).

\section{ACKNOWLEDGMENTS}

E.Q. and A.A. thank Dr. Gwilherm Kerherve for his help with the XPS system, and Dr. Peter Klusener, Dr. Samuel J. Cooper and Prof. Nigel Brandon for fruitful discussions. I.D.S acknowledges the Imperial College Research Computing Service (10.14469/hpc/2232), and associated support services used during this work. This work has been funded by the Engineering and Physical Sciences Research Council (EPSRC/17100026 and EPSRC/R002010/1 grants), the European Commission (grant FETPROACT-2018-2020 "HARVERSTORE" 824072) and Shell Global Solutions B.V.

\section{AUTHOR CONTRIBUTIONS}

E.Q. and A.A. conceived the research plan. I.S. carried out the computational work. A.C. conducted the LEIS analysis. Q.M. assisted in the NZSP synthesis and collected part of the electrochemical measurements. E.Q. conducted the XPS analysis and collected all remaining data. A.A. supervised the project. All the authors have contributed to discussions, manuscript preparation, and revisions leading to the final format of the manuscript. 


\section{DECLARATION OF INTERESTS}

The authors declare no competing interests. 